CMRO 03 (10), 665-681 (2020)

ISSN (O) 2589-8779 | (P) 2589-8760

CLINICAL TRIAL

\title{
Efficacy and Safety of The Herbal Combination Containing Tropaeoli Majoris Herba and Armoraciae Rusticanae Radix in Patients Suffering from Uncomplicated, Acute Rhinosinusitis: A Randomized, Double-Blind, Placebo Controlled, Two-Arm, Parallel Group, Phase IV Clinical Trial
}

\section{Uwe Albrecht $^{1^{*}} \mid$ Rainer Stange $^{2} \mid$ Nadine Schermuly $^{3}$}

\footnotetext{
${ }^{1}$ Mediconomics GmbH, Hannover, Germany

${ }^{2}$ Internal and Complementary Medicine, Immanuel

Krankenhaus Berlin-Wannsee, Berlin, Germany

${ }^{3}$ Mediconomics GmbH, Hannover, Germany,
}

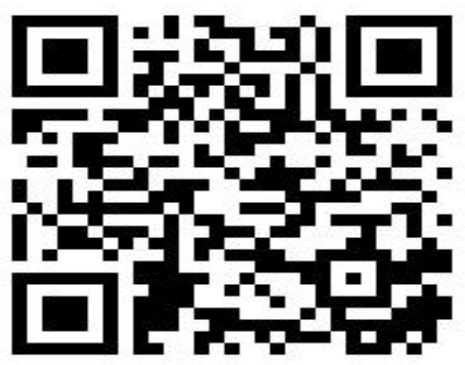

\section{Abstract}

Objectives: Demonstration of the efficacy and safety of an herbal combination containing horseradish root and nasturtium herb for the treatment of uncomplicated, acute rhinosinusitis. Design: Randomized, double-blind, placebo-controlled, two-arm, parallel group, multicenter, phase IV clinical study. Setting: Sixteen centers in Germany, from October 2017 to March 2018. Participants: Adult patients ( $\geq 18-75$ years, male and female) diag-nosed with uncomplicated, acute rhinosinusitis. Interventions: Participants received the herbal combination or placebo ( 3 x 4 per day) for 14 days.

Clinical trial registration: EudraCT 2017-002081-40.

Endpoints: Primary endpoint was $\mathrm{MRSS}_{i n v} / \mathrm{MRSS}_{p a t}$ documented between day 6 and day 10, computed as AUC, assessed by ANCOVA with day 3 as covariate. $\mathrm{MRSS}_{i n v} / \mathrm{MRSS}_{\text {pat }}$, responder rates, efficacy assessment and SNOT-20 GAV were used as secondary endpoints. Results: Out of 380 randomized participants, 238 were included in the FAS for statistical analysis. Treatment with the herbal combination revealed a significant smaller AUC (14.99) compared to placebo (18.52, $\mathrm{p}=0.0003)$. Moreover, $\mathrm{MRSS}_{i n v} / \mathrm{MRSS}_{\text {pat }}$ was significantly lower after administration of the herbal combination in comparison to placebo (3.60 herbal combination vs. 4.40 placebo, $\mathrm{p}=0.0018$ ). Responder rates were significantly higher at visit 3 of patients receiving the herbal combination compared to placebo (92.1\% herbal combination vs. $83.3 \%$ placebo, $\mathrm{p}=0.0418$ ). Adverse events occurred in $21.9 \%$ and $18.6 \%$ of participants receiving the herbal combination and placebo, respectively. Most common adverse events were headaches and gastrointestinal complaints.

Conclusion: Efficacy and safety of the herbal combination in comparison to placebo were confirmed while the treatment was well tolerated. Keywords: Uncomplicated, acute rhinosinusitis, herbal medicinal product, MRSS, SNOT-20, horseradish, nasturtium herb, randomized clinical trial, Tropaeoli majoris herba, Armoraciae rusticanae radix 


\section{1 | INTRODUCTION}

$\mathrm{R}$ hinosinusitis (RS) is defined as simultaneous inflammation of the nasal mucous membrane ("rhinitis") and inflammation of the mucous membrane of the paranasal sinuses ("sinusitis"), which leads to symptoms like nasal blockage, obstruction or congestion or nasal discharge (anterior, posterior, nasal drip). Additional symptoms include facial pain or pressure, the reduction or loss of smell, headache and fever $(1,2)$.

Complicated RS is characterized by intracranial and intraorbital spread of infection, which is not observed in case of uncomplicated RS $(3,4)$. Acute RS (ARS) is defined if symptoms occur for less than 12 weeks, while patients showing symptoms for more than 12 weeks are diagnosed with chronic RS (CRS) (1, 2). ARS is further divided into bacterial and viral ARS. The latter is often caused by viruses such as rhinovirus, adenovirus and parainfluenza virus and represents the most common cause of ARS. Over time, acute viral RS (AVRS) may evolve into acute bacterial RS (ABRS), which is often caused by Streptococcus pneumonia, Haemophilus influenza and Moraxella catarrhalis (5) .

There are several guidelines for the definition, diagnosis and treatment of RS, which partly differ regarding their criteria for diagnosis. However, nasal congestion, obstruction or blockage, purulent rhinorrhea and facial pain or pressure are considered as primary indicators for the diagnosis of ARS (6). Additionally, markers of inflammation such as CReactive Protein (CRP), Procalcitonin or Erythrocyte Sedimentation Rate (ESR) support ARS diagnosis. Further assessment is not necessary even though techniques such as nasal culture or sinus puncture may be used to analyze specific pathogens involved in ARS. In case of severe ARS, imaging techniques like CT (computed tomography) are considered (7) .

With affecting 6 to $15 \%$ of the population, ARS is a very common condition and thus, causes significant direct and indirect costs. The former involves costs due to prescriptions, visits and imaging techniques while employees suffering from ARS indirectly create costs due to absence and reduced productivity (7)
Since ARS resolves over time, antibiotics are not necessary and should only be considered in case of severe ARS. Instead symptomatic treatment represents the primary therapy option including intranasal corticosteroids, saline nasal irrigation and analgesics (5). Especially herbal drugs are often used to treat ARS symptoms. However, there are only few double-blind randomized clinical studies investigating the efficacy of herbal drugs (7) .

The drug under investigation is licensed in Germany since 2005 and represents a combination herbal medicinal product (CHMP), which contains powdered nasturtium (Tropaeoli majoris herba) and horseradish root (Armoraciae rusticanae radix) as active pharmaceutical ingredients (API). Efficacy and safety were demonstrated for the prophylactic treatment of episodes of respiratory tract infections (8). In addition, previous findings indicate that the herbal combination represents an alternative to antibiotics for the treatment of urinary tract infection (9) and especially acute, uncomplicated cystitis (10). Previously, an observational study had shown that the herbal combination was as effective in treating acute sinusitis as antibiotics $(11,12)$.

However, there is still insufficient evidence regarding the efficacy of horseradish root and nasturtium for the treatment of ARS. Therefore, this doubleblind randomized, placebo-controlled, two-arm, parallel group, phase IV clinical study aimed to investigate the efficacy and safety of the herbal combination in adult patients suffering from uncomplicated ARS.

\section{2 | MATERIAL AND METHODS}

\section{Study design:}

Supplementary information The online version of this article (https://doi.org/10.15520/jcmro.v3i10.35 0) contains supplementary material, which is available to authorized users.

Corresponding Author: Uwe Albrecht

Misburger Straße 81B, 3065 Hannover, Germany, Phone: +49 (0)5115609980,

Email:albrecht@mediconomics.com 


\section{RANDOMIZED CONTROLLED TRIAL OF AN HERBAL COMBINATION CONTAINING TROPAEOLI MAJORIS HERBA AND ARMORACIAE RUSTICANAE RADIX}

This was a multicenter, randomized, double-blind, placebo-controlled, two-arm, parallel group, phase IV clinical study, which was conducted from October 2017 to March 2018 in 16 study sites in Germany. The study protocol was approved by the leading Ethics Committee "Landesamt für Gesundheit und Soziales Berlin" (LAGeSo; Berlin, Germany) on September 05, 2017 (EudraCT-number: 2017002081-40). The clinical trial has been conducted in accordance with the requirements of the study protocol and in compliance with the ethical principles of the Guidelines for Good Clinical Practice (GCP), the Declaration of Helsinki and in strict compliance with the German Drug Law and the Federal Data Protection Act. The aim of this study was to investigate the safety, efficacy and tolerability of the herbal combination in comparison to placebo for the treatment of uncomplicated, acute rhinosinusitis.

Randomization of participants was conducted by an independent biostatistician who was not involved in patient recruitment and data collection. Similar appearance of the herbal combination and placebo with no information on the blisters regarding designation and strength of study medication ensured blinding of the investigator and patient. The allocation of patient and study medication was based on the randomization number on the blister and the randomization list.

Study medication was provided by the sponsor and packaged for the purpose of this study. All persons involved in the study, including monitor, principal investigator, biometrician and sponsor remained blinded throughout the study. In case of emergency, subjects could be unblinded at any time. Final unblinding was performed by the biometrician after Blind Data Review and closure of the database.

\section{Participants:}

Outpatients suffering from uncomplicated, acute rhinosinusitis between 18 and 75 years of age were enrolled. To assess manifestation of ARS, a major rhinosinusitis symptom score (MRSS) was used by investigators $\left(\mathrm{MRSS}_{i n v}\right)$ and patients $\left(\mathrm{MRSS}_{\text {pat }}\right)$. Patients were included in the study if they got diagnosed with (recurrent) uncomplicated, acute rhinosinusitis, a $\mathrm{MRSS}_{i n v}$ of $\geq 8$ and $\leq 12$ along with nasal obstruction (blocked nose) and facial pain or pressure (mild or moderate). Moreover, symptoms had to occur no later than 3 days prior to inclusion in the study.

Exclusion criteria comprised the following: chronic rhinosinusitis; polyposis nasi; cystic fibrosis; anatomical changes of nasal septum; acute symptoms of allergic rhinitis (hayfever); clinically relevant findings (significant changes in comparison to expected values); patients suffering from asthma; known intolerability against powdered nasturtium, horseradish root or any excipient of the herbal combination; patients with contraindications listed in the patient information; immunosuppressed patients; patients showing signs of fulminant, bacterial sinusitis; severe diseases of liver or kidneys; severe somatopathic, neurological and/or psychiatric disease; diseases or situations, which put the patient at risk or affect study results; alcohol or drug abuse. Moreover, patients with the following medication were excluded: intake of immunosuppressive drugs within the last 8 weeks prior to screening; systemic or nasal antibiotics or corticosteroids within 30 days before inclusion; systemic, antiviral treatment including Aciclovir, Zanamivir or Oseltamivir during the last 30 days prior to visit 1; intake of homeopathic for symptomatic treatment of a common cold or drugs with immunomodulatory characteristics within 7 days prior inclusion; patients who need antibiotic treatment at the time of inclusion. Additionally, patients were excluded in case of participation in other clinical studies or the same study within 6 weeks prior to inclusion or during this clinical trial. Moreover, pregnancy; lactation or use of unsafe contraceptive represented additional exclusion criteria.

\section{Interventions:}

One tablet of the herbal combination contains 80 mg horseradish root powder and $200 \mathrm{mg}$ nasturtium powder, produced by grinding of unmodified dried plants. Placebo tablets were provided by the manufacturer of the herbal combination and resembled the drug under investigation. Participants of each group were instructed to take 4 tablets 3 times per day for 14 days starting at visit 1 (day 0 ). In case of insufficient efficacy, applying physical heat and oral Paracetamol as medicinal product was used as rescue treatment. 


\section{Outcome measures:}

In this study, a co-primary endpoint represented the primary endpoint, which was the mean of MRSS $_{i n v}$ and $\mathrm{MRSS}_{\text {pat }}\left(\mathrm{MRSS}_{\text {inv }} / \mathrm{MRSS}_{\text {pat }}\right)$ between day 6 and day 10, computed as AUC (area under the curve) and assessed by ANCOVA (analysis of covariance) with day 3 as covariate. A 4-point scale was used $(0=$ no symptoms, $1=$ mild symptoms, $2=$ moderate symptoms, $3=$ severe symptoms) to evaluate manifestation of ARS symptoms (anterior and posterior secretion, nasal congestion, headache, and facial pain/pressure), resulting in a maximum MRSS of 15 . On the one hand, symptoms were assessed by the investigator $\left(\mathrm{MRSS}_{i n v}\right)$ at each visit. On the other hand, symptoms were evaluated on a daily basis by the patient $\left(\mathrm{MRSS}_{p a t}\right)$ and documented in the patients' diary.

Secondary endpoints included the AUC of $\mathrm{MRSS}_{i n v}$ and $\mathrm{MRSS}_{\text {pat }}$. Additionally, changes of the SinoNasal-Outcome Test - German Adapted Version (SNOT-20 GAV) total score and the sub scores primary nasal symptoms (PNS) and secondary rhinogenic symptoms (SRS) were analyzed. Moreover, responder rates and evaluation of efficacy were used as secondary outcome measures. Efficacy was assessed by the investigator at visit 2-5 in comparison to baseline at visit 1 based on the following scale: $0=$ symptoms diminished; $1=$ symptoms improved; $2=$ no change of symptoms; $3=$ symptoms worsened. Patients whose symptoms improved or diminished (scores 0 and 1) were defined as responder whereas symptoms of non-responder did not change or worsened (scores 2 and 3). For evaluation of safety and tolerability, adverse events, vital signs (blood pressure, pulse and body temperature), clinical chemistry and histopathology were documented. Moreover, tolerability was assessed at visit 5 by the investigator and patient using a 11-point visual analogue scale $(10=$ best tolerability; $0=$ worst tolerability $)$.

\section{Procedure:}

This study covered a period of 14 days Figure 1 , during which 5 visits took place, 2 of which were conducted on the phone (visit 2 and 4).

At visit 1, patients in an outpatient treatment were screened and checked regarding inclusion and exclusion criteria. After informed consent was ob- tained, demographic, anamnestic data and concomitant diseases, intake of concomitant medication and medication taken within the last 30 days as well as baseline MRSS $_{i n v}$ and MRSS $_{p a t}$ were recorded. Moreover, vital signs were checked, a pregnancy test and physical examination were conducted. Suitable participants were randomized and received study medication and a patient's diary. Additionally, the SNOT-20 GAV questionnaire was completed in the presence of the investigator at visits 1, 3 and 5 .

Inclusion and exclusion criteria, concomitant diseases and medication, vital signs, $\mathrm{MRSS}_{i n v}$ and $\mathrm{MRSS}_{\text {pat }}$, adverse events (AE) and severe adverse events (SAE) were checked at each visit. At visits 1 , 3 and 5, blood samples were taken for clinical chemistry. Efficacy of study medication was evaluated by the investigator at visits 2, 3, 4 and 5. On a daily basis, participants documented $\mathrm{MRSS}_{\text {pat }}$, intake of study medication, rescue medication (Paracetamol) and rescue treatment (applying physical heat) as well as body temperature above $38.5^{\circ} \mathrm{C}$. $\mathrm{MRSS}_{\text {pat }}$ entries were checked by the investigator at visit 3 and 5. On a weekly basis, adverse events, changes of concomitant medication and non-medical treatment as well as the amount of horseradish root or nasturtium ingested with the diet were documented by the patient. At the end of the study, patients returned the diaries and remaining study medication.

\section{Statistical analysis:}

Sample size calculation and statistical analyses were carried out in accordance with the ICH-GCP guidelines. For sample size calculation, a $\mathrm{MRSS}_{i n v}$ of $3.4 \pm 3.3$ and $2.4 \pm 2.5$ and a MRSS $_{p a t}$ of $3.5 \pm 3.3$ and $2.6 \pm 2.9$ was assumed for placebo and herbal combination, respectively. To detect a difference between the herbal combination and placebo, a power of $93 \%$ for $\mathrm{MRSS}_{i n v}$ and $80 \%$ for $\mathrm{MRSS}_{\text {pat }}$ as well as a sample size of 190 participants per group was required.

If initial data was missing, NOCB (next observation carried backward) was used for data imputation while missing intermediate or final values were imputed using LOCF (last observation carried forward). Additionally, a sensitivity analysis (SENS) was performed, in which missing data was imputed using NOCB, interpolation and linear regression. 


\section{RANDOMIZED CONTROLLED TRIAL OF AN HERBAL COMBINATION CONTAINING TROPAEOLI MAJORIS HERBA AND ARMORACIAE RUSTICANAE RADIX}

Efficacy was evaluated using mixed model repeated measurement (MMRM) covariance analysis, ANCOVA and $\chi^{2}$-test. Evaluation of tolerability and adverse events was performed with $\chi^{2}$-test and $\mathrm{U}$ test while changes of vital signs were analyzed using the Wilcoxon-Mann-Whitney U-Test and t-test. All tests used a level of significance of $\alpha=0.05$ (twosided).

Based on the exclusion criterion allergic RS, patients likely suffering from allergic RS had to be taken into account. The pollen flight causing allergic RS is mainly characterized by alder and hazel pollen, which reached its peak in February and March 2018. Therefore, patients enrolled in the study during these months were excluded from the FAS, which was used for evaluation of efficacy.

\section{Results}

Three hundred eighty-seven subjects were screened, of which 380 patients were randomized Figure 2 . The ITT population, which comprised patients with at least one post-baseline value, included 185 patients, each receiving the herbal combination or placebo (Figure 2 ITT). Due to allergic RS, 67 patients and 65 patients were excluded from the FAS in the herbal combination and placebo group, respectively (Figure 2 FAS). The FAS included 118 patients receiving the herbal combination while 120 patients received placebo. Patients with no severe protocol violation represented the PP population in which 110 patients administered the herbal combination and 114 patients received placebo. Demographic data of patients in the FAS was homogeneous Table 1.

The number of patients (n) and their proportion in the corresponding treatment group (\%) are given. Abbreviations: ARS: acute rhinosinusitis, BMI: body mass index, FAS: full-analysis-set

Baseline data was documented at day 0, which revealed a median $\mathrm{MRSS}_{i n v}$ and $\mathrm{MRSS}_{p a t}$ of 9 in both groups Table 2. Additionally, compliance was recorded and defined if $\geq 85 \%$ of study medication was used. Both groups of the FAS population revealed a compliance rate of $100 \%$. Only one subject of the FAS population revealed a compliance rate $<85 \%$. Consequently, a homogenous population was evaluated.
On day 0 , visit 1 , the investigator and patient evaluated the manifestation of the 5 main symptoms of ARS. The number of patients (n) and their proportion (\%) in the treatment groups are depicted. Abbreviations: MRSS $_{\text {inv }}$ : major rhinosinusitis symptom score, assessed by the investigator, $\mathrm{MRSS}_{\text {pat }}$ : major rhinosinusitis symptom score, assessed by the patient.

\section{Efficacy:}

The intensity of symptoms, indicated as $\mathrm{MRSS}_{\text {inv }} / \mathrm{MRSS}_{\text {pat }}$, revealed a difference between placebo and the herbal combination after day 6. An obvious difference for the endpoint $\mathrm{MRSS}_{\text {inv }} / \mathrm{MRSS}_{\text {pat }}$ was shown between day 6 and day 10 whereby administration of the herbal combination resulted in a lower $\mathrm{MRSS}_{i n v} / \mathrm{MRSS}_{\text {pat }}$ compared to placebo Figure 3. After 10 days, $\mathrm{MRSS}_{i n v} / \mathrm{MRSS}_{p a t}$ decreased further but a marked difference between the groups was no longer observed Figure 3.

A previous study showed that the herbal combination product exhibited a delayed onset of activity. Therefore, day 3 was used as baseline for further evaluation of the treatment period between day 6 and day 10 Figure 4. On day 6, participants of the phytotherapeutic group showed a lower $\mathrm{MRSS}_{i n v} / \mathrm{MRSS}_{\text {pat }}$ with 4.44 in contrast to subjects of the placebo group with 5.19 (Figure $4 \mathrm{~A}, \mathrm{p}=0.0070$ ). The most significant effect $(\mathrm{p}=0.0002)$ was observed on day 7 as $\mathrm{MRSS}_{\text {inv }} / \mathrm{MRSS}_{\text {pat }}$ was 3.92 in patients treated with the herbal combination, whereas participants receiving placebo showed a $\mathrm{MRSS}_{i n v} / \mathrm{MRSS}_{\text {pat }}$ of 4.95 ( Figure $4 \mathrm{~A}$ ). On day $10, \mathrm{MRSS}_{i n v} / \mathrm{MRSS}_{\text {pat }}$ revealed a slight difference between placebo and phytotherapeutic group (Figure $4 \mathrm{~A}$ ). The mean of $\mathrm{MRSS}_{i n v} / \mathrm{MRSS}_{\text {pat }}$ between day 6 and day 10, showed a significant difference in favor of the herbal combination product (Figure $4 \mathrm{~B}, \mathrm{p}=0.0018$ ). Importantly, the AUC of $\mathrm{MRSS}_{i n v} / \mathrm{MRSS}_{\text {pat }}$ was significant lower after administration of the herbal combination in comparison to placebo (Figure $4 \mathrm{C}$, $\mathrm{p}=0.0003$ ).

Assessment of efficacy by the investigator (at visit 3) revealed that $92.1 \%$ of patients receiving the herbal combination showed improved symptoms or even no more symptoms whereas a lower proportion of 
TABLE 1: Demographic and anamnestic data of the FAS.

\begin{tabular}{|c|c|c|c|c|c|c|}
\hline \multirow[t]{2}{*}{ Variable } & \multicolumn{2}{|c|}{$\begin{array}{l}\text { Herbal } \\
\text { combina- } \\
\text { tion }\end{array}$} & \multicolumn{2}{|c|}{ Placebo } & \multicolumn{2}{|l|}{ Total } \\
\hline & & $\%$ & & $\%$ & & $\%$ \\
\hline Patients diagnosed with ARS & 118 & & 120 & & 238 & \\
\hline Median age (years) & 33.0 & & 37.0 & & 36.0 & \\
\hline$\leq 30, n, \%$ & 50 & 42.2 & 44 & 36.7 & 94 & 39.5 \\
\hline $31-60, n, \%$ & 64 & 54.2 & 69 & 57.5 & 133 & 55.9 \\
\hline$>60, n, \%$ & 4 & 3.4 & 7 & 5.8 & 11 & 4.6 \\
\hline Male, $n, \%$ & 35 & 29.7 & 42 & 35.0 & 77 & 32.4 \\
\hline Female, $n, \%$ & 83 & 70.3 & 78 & 65.0 & 161 & 67.6 \\
\hline Median BMI (kg/m2) & 25.6 & & 25.6 & & 25.6 & \\
\hline \multicolumn{7}{|c|}{$\begin{array}{l}\text { Time since onset of acute symptoms prior } \\
\text { to enrollment in the study (days) }\end{array}$} \\
\hline 0 & 2 & 1.7 & 4 & 3.3 & 6 & 2.5 \\
\hline 1 & 35 & 29.7 & 41 & 34.2 & 76 & 31.9 \\
\hline 2 & 77 & 65.3 & 69 & 57.5 & 146 & 61.3 \\
\hline 3 & 4 & 3.4 & 6 & 5.0 & 10 & 4.2 \\
\hline \multicolumn{7}{|c|}{$\begin{array}{l}\text { Known, relevant previously treated dis- } \\
\text { eases and/or medical interventions }\end{array}$} \\
\hline no & 85 & 72.0 & 95 & 79.2 & 180 & 75.6 \\
\hline yes & 33 & 28.0 & 25 & 20.8 & 58 & 24.4 \\
\hline \multicolumn{7}{|c|}{$\begin{array}{l}\text { Concomitant diseases at the beginning of } \\
\text { the study }\end{array}$} \\
\hline no & 63 & 53.4 & 63 & 52.5 & 126 & 52.9 \\
\hline yes & 55 & 46.6 & 57 & 47.5 & 112 & 47.1 \\
\hline
\end{tabular}

TABLE 2: Baseline MRSS $_{i n v}$ and MRSS $_{p a t}$.

\begin{tabular}{llllllllll} 
& \multicolumn{2}{l}{$\begin{array}{l}\text { MRSSinv } \\
\text { Herbal } \\
\text { combi- } \\
\text { nation }\end{array}$} & Placebo & \multicolumn{3}{l}{$\begin{array}{l}\text { MRSSpat } \\
\text { Herbal } \\
\text { combi- } \\
\text { nation }\end{array}$} & Placebo \\
\hline$<8$ & - & - & - & - & 24 & 20.3 & 29 & 24.2 \\
\hline $8-12$ & 118 & 1 & 120 & 1 & 89 & 75.4 & 88 & 73.3 \\
\hline$>8$ & - & - & - & - & 5 & 4.2 & 3 & 2.5 \\
\hline Median & 9.0 & & 9.0 & & 9.0 & & 9.0 & \\
\hline
\end{tabular}




\section{RANDOMIZED CONTROLLED TRIAL OF AN HERBAL COMBINATION CONTAINING TROPAEOLI MAJORIS HERBA AND ARMORACIAE RUSTICANAE RADIX}

subjects in the placebo group revealed improved or no more symptoms $(83.3 \%$, Figure 5 A). Thus, a treatment with the herbal combination resulted in a significant higher rate of responders at visit 3 (Figure $5 \mathrm{~B}, \mathrm{p}=0.0418$ ).

A significant decrease of the total SNOT-20 GAV was observed (Figure $6 \mathrm{~A}, \mathrm{p}=0.0351$ ) in the phytotherapeutic group while at visit 3 symptoms of the sub scores PNS and SRS improved significantly after administration of the herbal combination compared to placebo (Figure 6 A, PNS: $p=0.0427$, SRS: $p=0.0128$ ). Importantly, administration of the herbal combination resulted in a greater reduction of the symptoms anterior secretion $(p=0.0235)$ and headache $(\mathrm{p}=0.0486)$ at visit 3 (Figure 6 B). Especially PNS, SRS, headache and anterior secretion are often experienced as the most troublesome symptoms by patients.

Taken together, these results demonstrate, that the herbal combination leads to a faster relief of ARS symptoms until day 10 , which improves quality of the patients' lives.

\section{Safety:}

Side effects occurred in 42 participants of the phytotherapeutic group $(21.9 \%)$ and in 35 patients receiving placebo (18.6\%) (Figure 7 A). The most commonly reported AE included headache (herbal combination: $7.8 \%$, placebo: $7.4 \%$ ) and gastrointestinal disorders (herbal combination: 5.7\%, placebo: $3.2 \%$ ). At least one possible causal link to study medication was observed in about $4 \%$ in both groups, whereby four patients developed gastrointestinal disorders. One patient of the placebo group required hospitalization due to visual field loss, which was reported as serious adverse event. However, its association with the study medication in the blinded phase was rated unlikely. Premature termination was necessary for six patients of both groups, which was mainly due to fever or infections. In terms of vital signs, hematology and clinical chemistry, no difference between placebo and the herbal combination was observed. Administration of placebo was better tolerated in comparison to the herbal combination. However, overall tolerability of the herbal combination was still rated with 8.73 and 8.82 on a 11-point scale by patients and investigator, respectively (Figure $7 \mathrm{~B}$ ).

\section{3 | DISCUSSION}

An effective treatment for uncomplicated, acute rhinosinusitis is of great interest since there is a high prevalence and the disease is associated with increased costs due to prescription of antibiotics, doctor's visits and absences due to sickness (7). ARS is often treated with antibiotics even though watchful waiting and symptomatic treatment are recommended while the use of antibiotics is considered in severe cases of ARS (5). Moreover, it is necessary to distinguish between ABRS and AVRS as antibiotics are ineffective in case of acute viral RS (3). Nevertheless, antibiotics are often prescribed to treat ARS despite of communicated severe concerns regarding overuse of antibiotics with increasing health care costs and dangerous antimicrobial resistance (13). Interestingly, results from a randomized controlled trial and a meta-analysis showed that a commonly prescribed antibiotic did not lead to significant improvement of ARS symptoms in comparison to placebo. In general, antibiotics were shown to have a rather small effect on recovery of ARS $(14,15)$.

Here we show that the herbal combination product resulted in less pronounced ARS symptoms $\left(\mathrm{MRSS}_{i n v} / \mathrm{MRSS}_{\text {pat }}\right.$ ) between day 6 and day 10 compared to placebo. Moreover, the total score of SNOT-20 GAV, PNS and SRS as well as individual symptoms such as anterior secretion and headache were significantly improved after administration of the herbal combination, which was verified by significantly higher responder rates at visit 3 in the phytotherapeutic group. Thus, administration of the herbal combination resulted in faster reduction of ARS symptoms in comparison to placebo, thereby improving quality of life of patients.

The MSS (Major symptom score) is widely used to evaluate efficacy in clinical trials (7). This study used a MRSS, which is based on the MSS and comprised the five most relevant ARS symptoms namely, anterior and posterior secretion, congested nose, headache and facial pain or pressure. In a similar study, the intensity of symptoms was only assessed 
by the investigator (16), while in this study the patients' perception (MRSS ${ }_{p a t}$ ) was additionally considered. Even though in another study MRSS $_{i n v}$ and MRSS $_{\text {pat }}$ were used, the symptom scores were evaluated separately (17). In contrast, the mean of both scores $\left(\mathrm{MRSS}_{i n v} / \mathrm{MRSS}_{p a t}\right)$ was additionally evaluated. Moreover, the AUC of $\mathrm{MRSS}_{\text {inv }} / \mathrm{MRSS}_{\text {pat }}$ represents an evaluation of a specific treatment period (day 6 - day 10), whereas other studies focused on specific time points $(16,17)$. Taken together, these endpoints consider both the investigators' and patients' assessment and provide information about the period of treatment instead of specific time points.

In the present study, $92.1 \%$ of patients were identified as responders at visit 3. Similar results for responder rates were reported by another study, which revealed $86.1 \%$ of patients as responders on day 7 (16). Interestingly, responder rates on day 10 and day 14 still showed significant differences between placebo and the herbal drug (16), which is surprising as spontaneous self-healing rates of $60-80 \%$ are observed after 2 weeks (2). It is therefore expected that the symptoms of both groups will be quite similar after 14 days, as demonstrated in this study. This ongoing effect shown by Jund et al. might be explained by patients suffering from allergic RS, as in this case no self-healing is involved. The results of the present study indicate that the herbal combination supports self-healing, resulting in a faster reduction of symptoms between day 6 and day 10 .

The efficacy of the herbal combination investigated in this study is likely based on the antimicrobial and antiviral activity of Tropaeoli majoris herba and Armoraciae rusticanae radix. Several findings revealed antibacterial, anti-oxidative and anti-inflammatory activity of nasturtium (18). In addition, it is likely that Tropaeoli majoris herba has antiviral characteristics (19). Moreover, it was reported that antimicrobial activity of the herbal combination is specific for $S$. pneumonia, $H$. influenza and $M$. catarrhalis. These properties were shown to be mediated by isothiocyanates, which are compounds of nasturtium and horseradish (20).

The safety profile of placebo and the herbal combination is nearly identical since adverse events in both groups were reported with similar incidence while placebo treatment was slightly better tolerated compared to the herbal combination product.

\section{4 | CONCLUSION}

In conclusion, daily intake of the herbal combination product enhances the relief of symptoms and thus improves quality of life of patients suffering from ARS while its use is safe and well tolerated.

\section{Acknowledgement}

This work was supported by Repha GmbH. Statistical analysis was performed by Dr. Joerg Schnitker, Institute for Applied Statistics, Bielefeld, Germany

\section{Disclosure}

Repha $\mathrm{GmbH}$ has not been involved in trial design, data collection and analysis. The authors report no conflicts of interest in this work.

\section{Data availability statement}

The data set is stored in a secured setting electronically by the CRO and in case of requirement data can be made available to CMRO.

The authors confirm that the data supporting the findings of this study are available within the article

\section{REFERENCES}

1. Fokkens RW, Lund V, Mullol J, et al. EPOS Pocket guide european position paper on rhinosinusitus and nasal polyps 2012. Rhinol Suppl. 2012;1-20.

2. Arbeitsgemeinschaft der Wissenschaftlichen Medizinischen Fachgesellschaften - Deutsche Gesellschaft für Hals-Nasen-Ohren- Heilkunde, Kopf- und Hals-Chirurgie e.V., Deutsche Gesellschaft für Allgemeinund Familienmedizin. Rhinosinusitis S2k-Leitlinie. 2017.

3. Rosenfeld RM, Piccirillo JF, Chandrasekhar $\mathrm{SS}$, et al. Clinical practice guideline (update): Adult sinusitis. Otolaryngol - Head Neck Surg. 2015;152:S1-S39. 
4. Kölln KA, Senior BA. Diagnosis and Management of Acute Rhinosinusitis. In: Thaler E, Kennedy DW, editors. Rhinosinusitis. New York: Springer-Verlag; 2008. p. 29-39.

5. Aring AM, Chan MM. Current concepts in adult acute rhinosinusitis. Am Fam Physician. 2016;94:97-105.

6. Meltzer EO, Hamilos DL. Rhinosinusitis diagnosis and management for the clinician: A synopsis of recent consensus guidelines. Mayo Clin Proc. 2011;86:427-443.

7. Fokkens WJ, Lund VJ, Mullol J, et al. EPOS 2012: European position paper on rhinosinusitis and nasal polyps 2012. A summary for otorhinolaryngologists. Rhinology. 2012;50:112.

8. Fintelmann V, Albrecht U, Schmitz G, et al. Efficacy and safety of a combination herbal medicinal product containing Tropaeoli majoris herba and Armoraciae rusticanae radix for the prophylactic treatment of patients with respiratory tract diseases: A randomised, prospective, double-blind, placebo-controlled phase III trial. Curr Med Res Opin. 2012;28:1799-1807.

9. Lau I, Albrecht U, Kirschner-Hermanns R. Phytotherapie bei katheter- assoziierten Harnwegsinfekten Beobachtungsstudie zur Wirksamkeit und Sicherheit einer fixen Kombination mit Kapuzinerkressenkraut und Meerrettichwurzel. [Phytotherapy in catheterassociated urinary tract infection: Observational study recording the efficacy and safety of a fixed herbal combination containing Tropaeoli majoris herba and Armoraciae rusticanae radix]. Urologe. 2018;57:1472-1480.

10. Stange R, Schneider B, Albrecht U, et al. Results of a randomized, prospective, doubledummy, double-blind trial to compare efficacy and safety of a herbal combination containing Tropaeoli majoris herba, Armoraciae rusticanae radix with co-trimoxazole in patients with acute, uncomplicated cystitis. Res Reports Urol. 2017;9:43-50.
11. Goos K-H, Albrecht U, Schneider B. Wirksamkeit und Verträglichkeit eines pflanzlichen Arzneimittels mit Kapuzinerkressenkraut und Meerrettich bei akuter Sinusitis, akuter Bronchitis und akuter Blasenentzündung im Vergleich $\mathrm{zu}$ anderen Therapien unter den Bedingungen der täglichen Praxis. [Efficacy and Safety Profile of a Herbal Drug containing Nasturtium herb and Horseradish Root in Acute Sinusitis, Acute Bronchitis and Acute Urinary Tract Infection in Comparison with Other Treatments in the Daily Practice]. Arzneimittel-Forschung/Drug Res. 2006;56:249-257.

12. Goos K-H, Albrecht U, Schneider B. Aktuelle Untersuchung zur Wirksamkeit und Verträglichkeit eines pflanzlichen Arzneimittels mit Kapuzinerkressenkraut und Meerrettich bei akuter Sinusitis, akuter Bronchitis und akuter Blasenentzündung bei Kindern im Vergleich zu anderen Antibiotika. [On-going Investigations on Efficacy and Safety Profile of a Herbal Drug Containig Nasturtium Herb and Horseradish Root in Acute Sinusitis, Acute Bronchitis and Acute Urinary Tract Infection in Children in Comparison with other Antibiotic Treatments]. Arzneimittel-Forschung/Drug

Res. 2007;57:238-246.

13. Sande MA, Gwaltney JM. Acute CommunityAcquired Bacterial Sinusitis: Continuing Challenges and Current Management. Clin Infect Dis. 2004;39:S151-S158.

14. Garbutt JM, Banister C, Spitznagel E, et al. Amoxicillin for Acute Rhinosinusitis. A Randomized Controlled Trial. Pharm Times. 2012;307:685-692.

15. Lemiengre $M$, van Drie Ml, Merenstein D, et al. Antibiotics for acute rhinosinusitis in adults. Cochrance Database Syst Rev. 2018; 9: CD006089. Published 2018 Sep 10.

16. Jund R, Mondigler M, Stammer H, et al. Herbal drug BNO 1016 is safe and effective in the treatment of acute viral rhinosinusitis. Acta Otolaryngol. 2015;135:42-50. 
17. Michalsen A, Palm J, Samer $H$, et al. Wirksamkeit und Verträglichkeit eines homöopathischen Komplexmittels (Sinusitis Hevert SL) bei akuter, unkomplizierter Rhinosinusitis: Eine multizen trische, randomisierte, doppelblinde, place bokontrollierte Studie an erwachsenen Patienten. [Efficacy and Safety of a Complex Homeopathic Remedy (Sinusitis Hevert SL) Used in Acute, Uncomplicated Rhinosinusitis: A Multicenter, Randomized, Double-Blind, Placebo - Controlled Trial in Adults]. Complement Med Res. 2017;24:139-146.

18. Butnariu M, Bostan C. Antimicrobial and antiinflammatory activities of the volatile oil compounds from tropaeolum majus 1 . (nasturtium). African J Biotechnol. 2011; 10:5900-5909.
19. Pulverer G. Benzylsenföl: Ein Breitbandantibiotikum aus der Kapuzinerkresse. [Benzyl mustard oil: A broad-spectrum antibiotic obtained from nasturtium]. Deutsche Medizinische Wochenschrift. 1968;98:16421649

20. Conrad A, Biehler D, Nobis T, et al. Broad spectrum antibacterial activity of a mixture of isothiocyanates from nasturtium (Tropaeoli majoris herba) and horseradish (Armoraciae rusticanae radix). Drug Res (Stuttg). 2013;63:65-68.
How to cite this article: Albrecht U., Stange R., Schermuly N. Efficacy and Safety of The Herbal Combination Containing Tropaeoli Majoris Herba and Armoraciae Rusticanae Radix in Patients Suffering from Uncomplicated, Acute Rhinosinusitis: A Randomized, Double-Blind, Placebo Controlled, Two-Arm, Parallel Group, Phase IV Clinical Trial. Journal of Current Medical Research and Opinion. 2020;665-680. https://doi.or $\mathrm{g} / 10.15520 /$ jemro.v3i10.350 
Treatment for 14 days

Placebo: $3 \times 4$ tablets / day

Herbal combination: $3 \times 4$ tablets / day

Visit

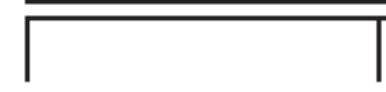

Day 0

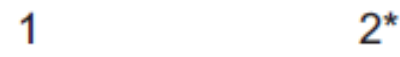

$2^{*}$

$3 \quad 7$

nterval

0
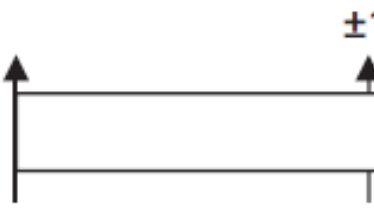

$\pm 1$

Daily documentation MRSS $_{\mathrm{p}}$
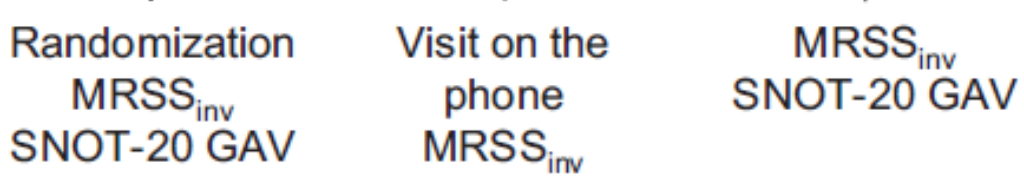

Visit on the phone

MRSS $_{\text {inv }}$

End of trial MRSS $_{\text {inv }}$ SNOT-20 GAV

FIGURE 1: Study design. This study was conducted within 14 days and included 5visits, with visit 2 and 4 performed on the phone. At each visit, MRSS ${ }_{i n v}$ was assessed by the investigator while MRSS $_{\text {pat }}$ was documented eachday in the patients' diary. At visit 1, 3 and 5, a SNOT-20 GAV questionnairewas answered. Patients were instructed to take 4 tablets of study medication 3times a day. MRSS $_{\text {inv }}$ : major rhinosinusitis symptom score, assessedby the investigator, MRSS $_{p a t}$ : major rhinosinusitis symptom score,assessed by the patient, SNOT-20 GAV: sino-nasal outcome test, german adaptedversion, 


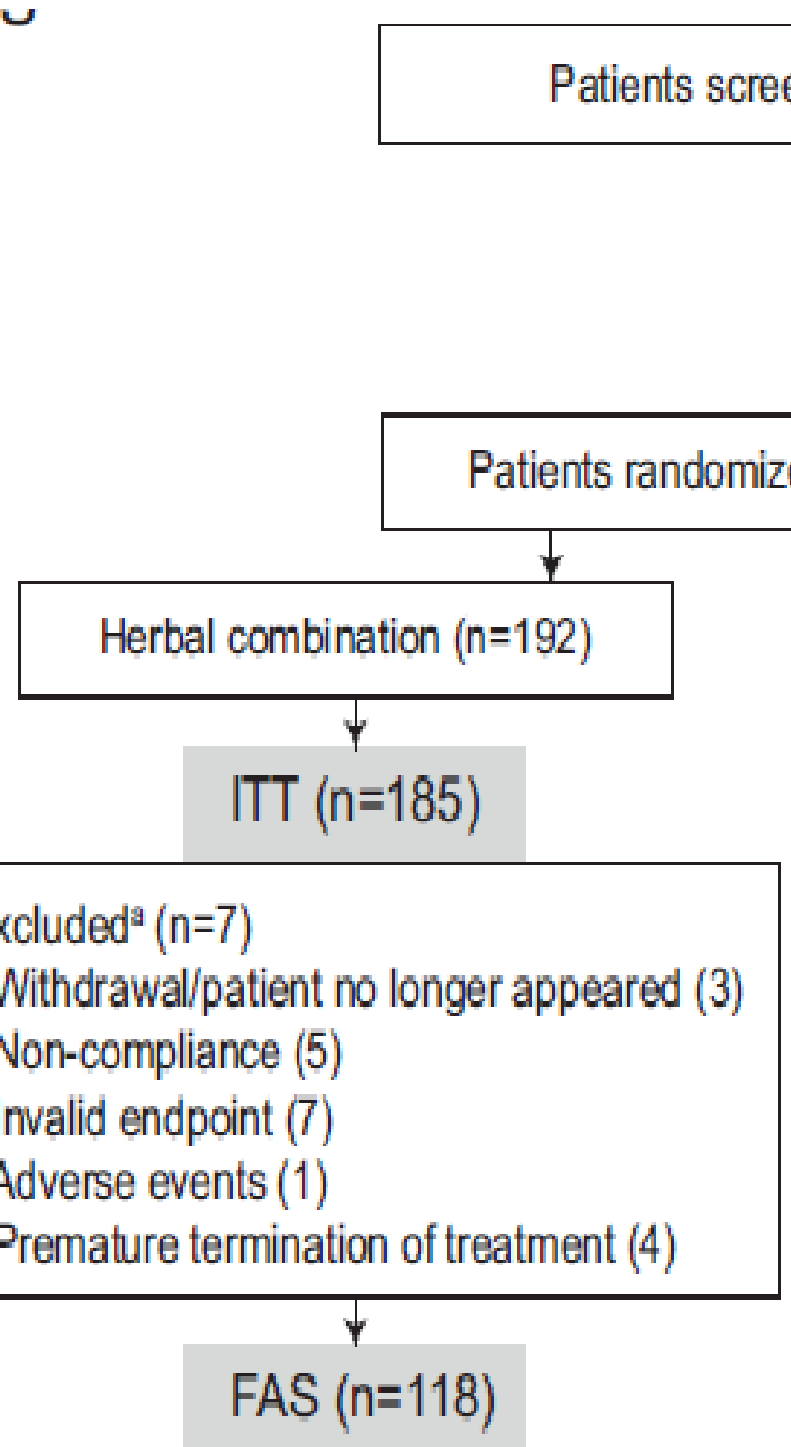

Screening failures/randomized ${ }^{\mathrm{a}}(\mathrm{n}=2)$ Screening failures/non-randomized ${ }^{a}(n=7)$

\section{Patients randomized and treated $(n=380)$}

\section{Excluded $^{a}(n=7)$}

- Withdrawal/patient nó longer appearéd (3)

- Non-compliance (5)

- Invalid endpoint (7)

- Adverse events (1)

- Premature termination of treatment (4)
Excludèd ${ }^{a}(n=67)$

- Allergic RS (67)

- Non-compliance (2)

- Adverse events (2)

- Forbidden concomitant medication (2)

- Premature termination of treatment (3)

\section{$\downarrow$}

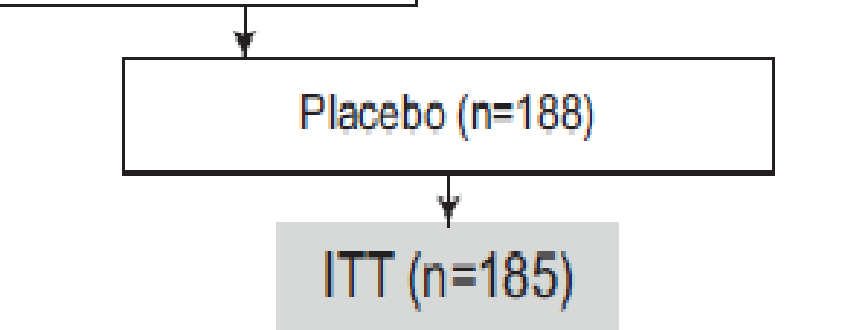

Excluded ${ }^{a}(n=7)$

- Withdrawal/patient no longer appeared (3)

- Non-compliance (5)

- Invalid endpoint (7)

- Adverse events (1)

- Premature termination of treatment (4)

FIGURE 2: Flow chart showing an overview of patientcollectives. FAS: full-analysis-set, ITT: intention-to-treat, PP: per protocol, ${ }^{a}$ : multiple answers possible 


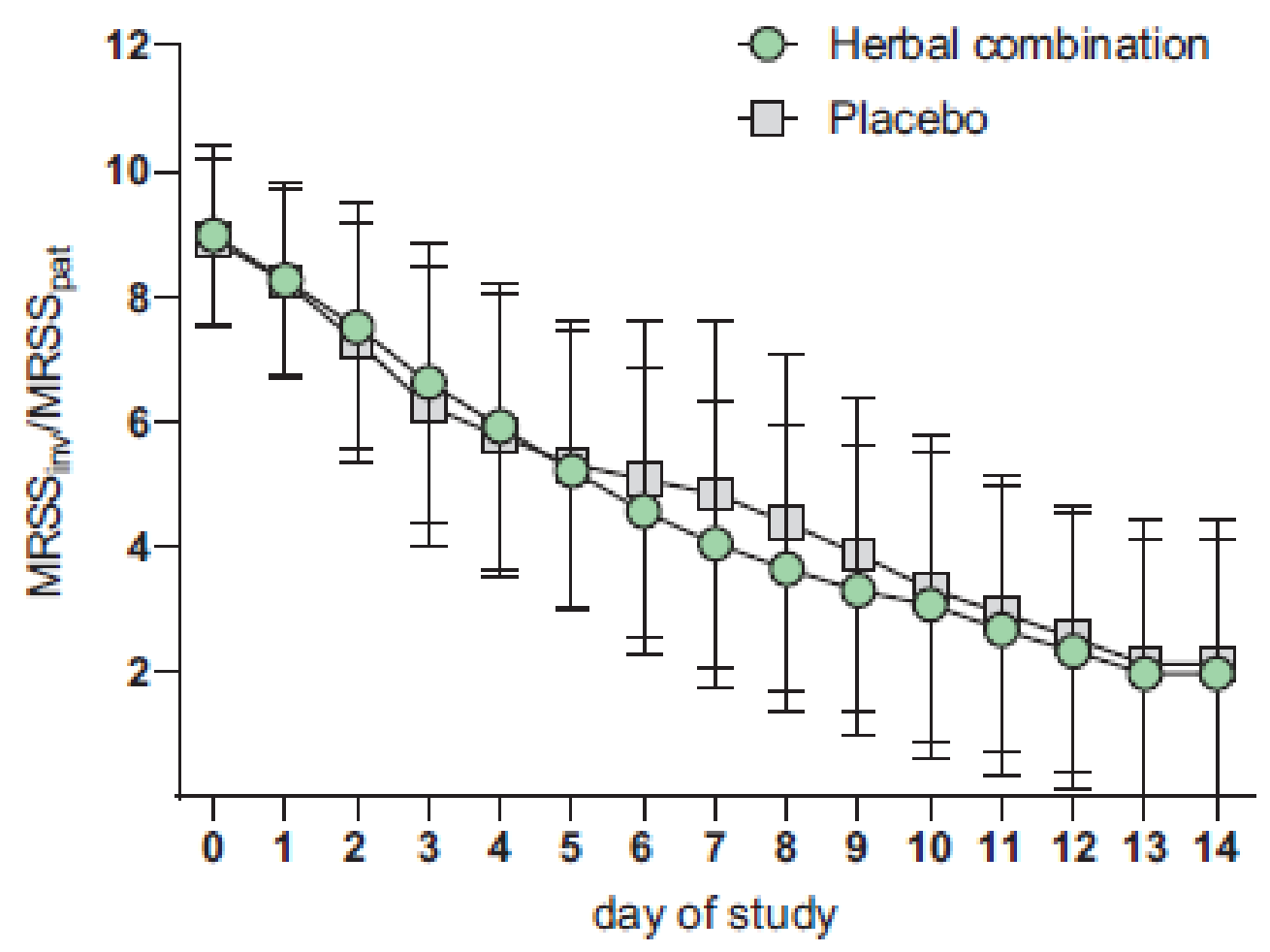

FIGURE 3: Efficacy of the herbal combination is observedbetween day 6 and day 10 of the treatment period. $\mathrm{MRSS}_{i n v} / \mathrm{MRSS}_{\text {pat }} \pm \mathrm{SD}$ between day 0 and day 14. MRSS ${ }_{i n v}$ : major rhinosinusitis symptomscore, assessed by the investigator, MRSS $_{\text {pat }}$ : major rhinosinusitissymptom score, assessed by the patient,

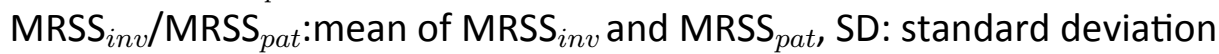



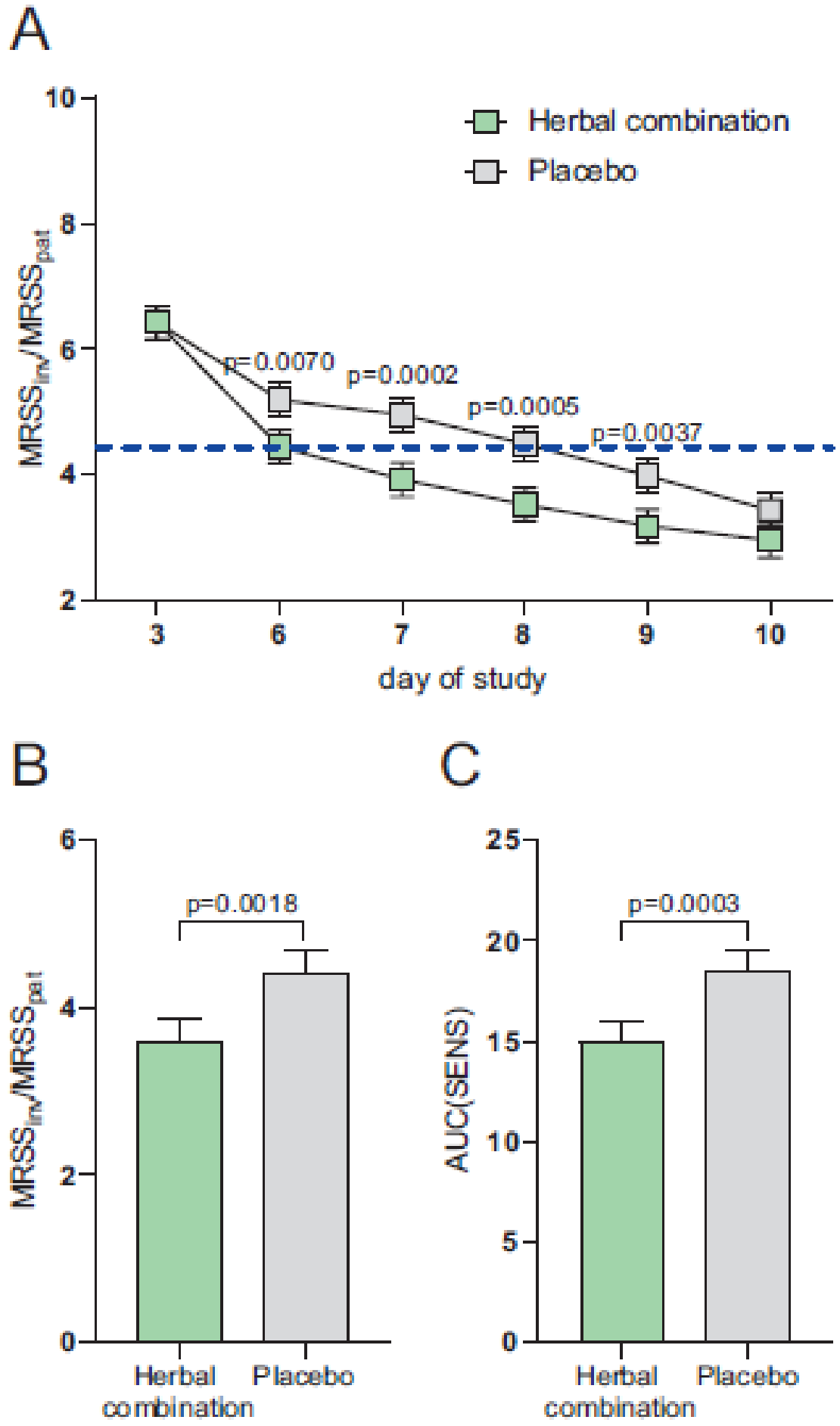

FIGURE 4: Treatment of ARS with the herbalcombination results in faster improvement of symptoms between day 6 and day 10compared to placebo. A MRSS ${ }_{i n v} / \mathrm{MRSS}_{\text {pat }} \pm \mathrm{SEM}$ between day6 and day 10, with covariate day 3. B LSmeans of MRSS ${ }_{i n v} /$ MRSS $_{p a t}$ between day 6 and day $10 \pm$ standard error. C AUC of MRSS $_{i n v} /$ MRSS $_{\text {pat }}$ between day 6 and day 10, with day 3 as baseline. ARS: acuterhinosinusitis, AUC: area under the curve, LSmeans: least square means, MRSS ${ }_{i n v}$ : major rhinosinusitis symptom score,assessed by the investigator, MRSS $_{\text {pat }}$ : major rhinosinusitissymptom score, assessed by the patient, MRSS $_{i n v} /$ MRSS $_{p a t}:$ mean of MRSS $i n v$ and MRSS $_{p a t}$, SEM: standard error of themean 
A
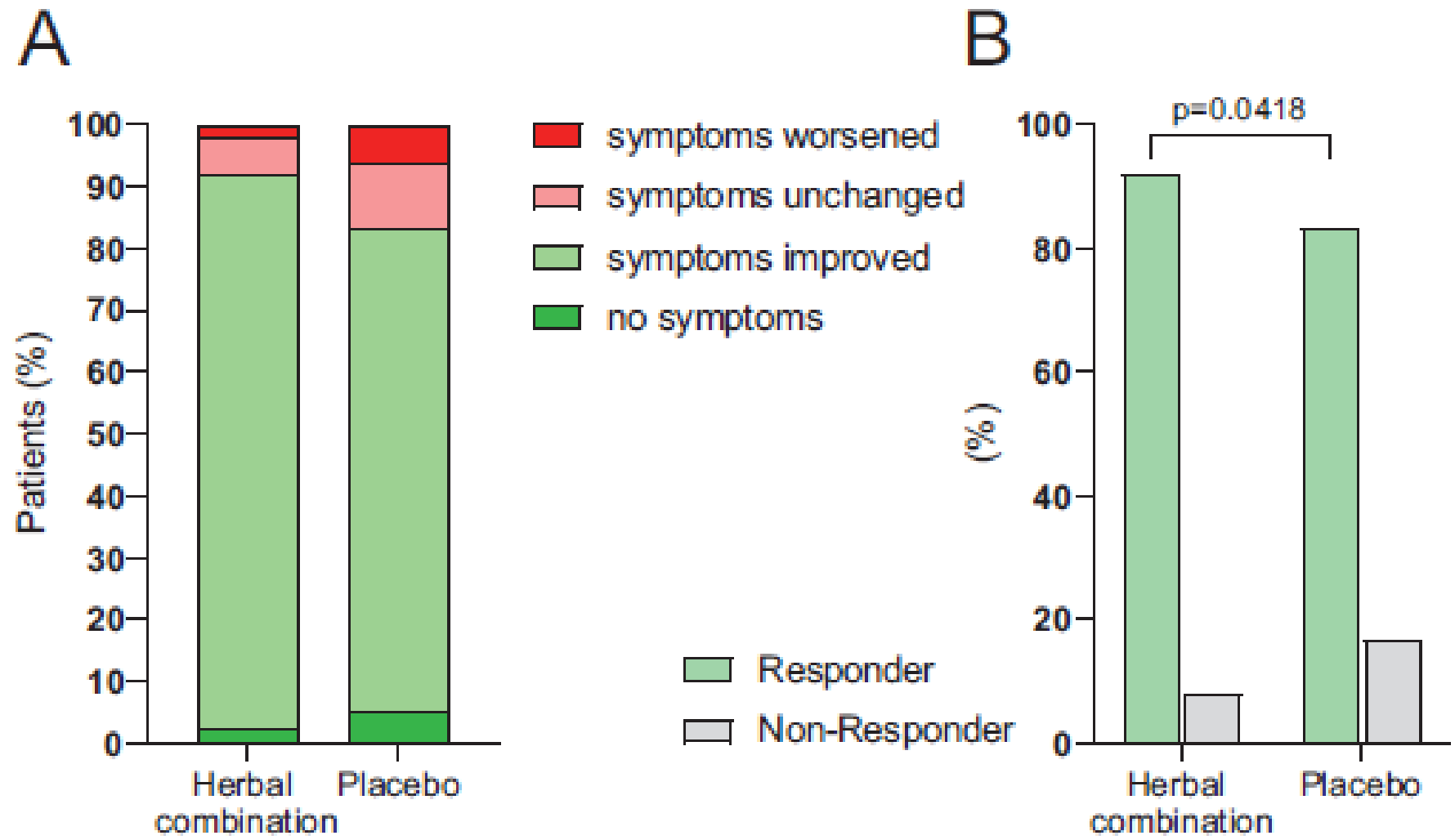

FIGURE 5: The herbal combination leads to ahigher amount of patients with improved or no more symptoms and higherresponder rates at visit 3 in comparison to placebo. A Efficacy of studymedication was assessed by the investigator at each visit compared to visit 1 . Theproportion of patients whose symptoms worsened, remained unchanged, improved ordiminished at visit 3. B Patients shown in A were categorized as responders ornon-responders. Patients with no symptoms or whose symptoms improved weredefined as responders while patients with worsened or unchanged symptoms weredefined as non-responders. 


\section{$A^{-}$}
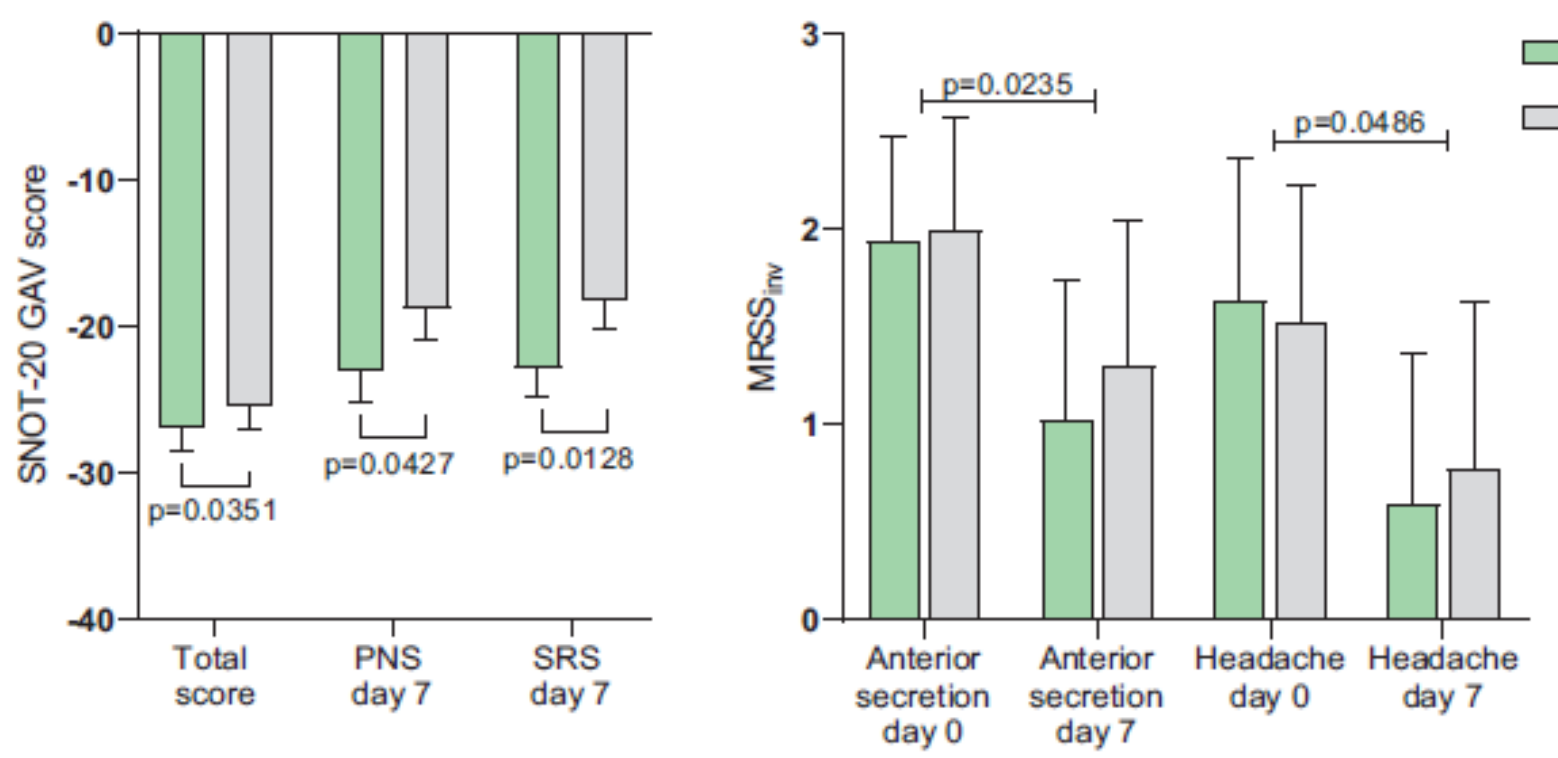

Herbal combination Placebo

FIGURE 6: Intake of the herbal combination results in agreater reduction of specific symptoms compared to placebo. A Change of theLSmeans of SNOT-20 GAV. LSmeans \pm SEM of the total score assessed at visit 3(day 7) and visit 5 (day 14) compared to baseline, sub scores PNS and SRSdocumented at visit 3 compared to baseline. B MRSS ${ }_{i n v}$ of thesymptoms anterior secretion and headache at visit 1 compared to visit 3. LSmeans:least square means, MRSS inv $_{\text {: }}$ major rhinosinusitis symptom score,assessed by the investigator, PNS: primary nasal symptoms, SEM: standard errorof the mean, SNOT-20 GAV: sino-nasal outcome test, german adapted version, SRS:secondary rhinogenic symptoms, V: visit 

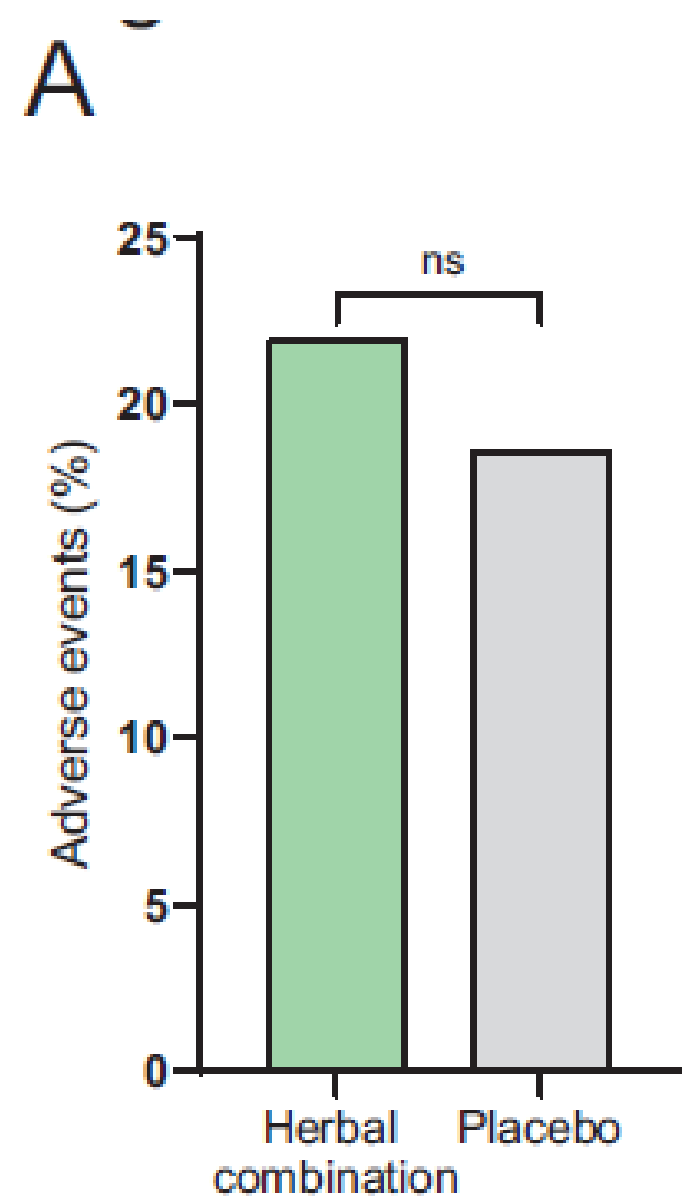
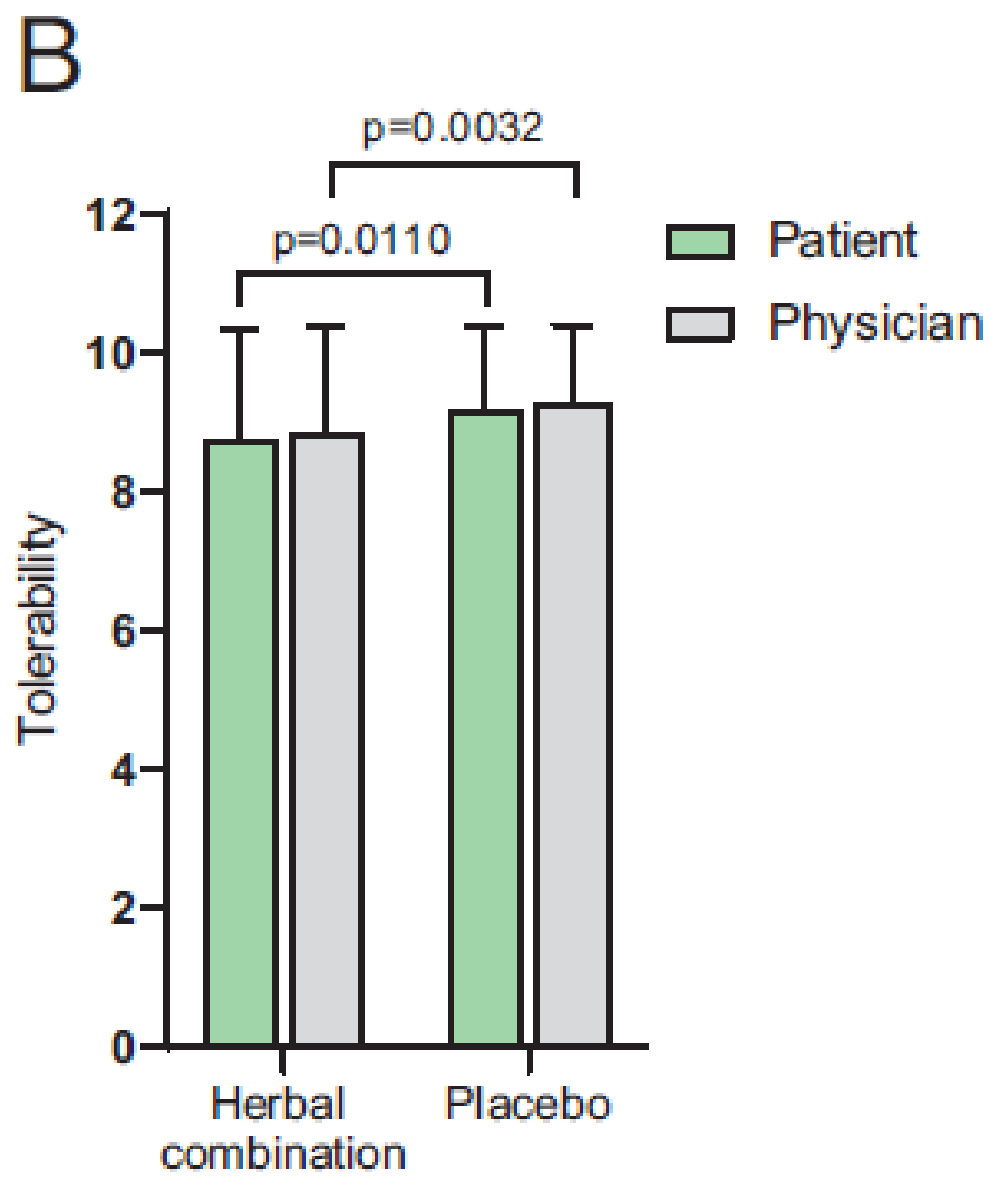

FIGURE 7: Use of the herbal combination was reported assafe and well tolerated. A Proportion of patients (\%), which showed adverseevents. B Tolerability was evaluated by the investigator and patient using an11-point visual analogue scale. The mean \pm SD of this evaluation is given. SD:standard deviation 